\title{
Improving the long-term adjustment of spinal cord injured persons
}

\author{
A Craig*,1, K Hancock ${ }^{2}$ and H Dickson ${ }^{3}$ \\ ${ }^{1}$ Department of Health Sciences, University of Technology, Sydney, Australia; ${ }^{2}$ Department of Health Sciences, \\ University of Technology, Sydney, Austalia; ${ }^{3}$ Aged Care and Rehabilitation, Bankstown Hospital NSW, Australia
}

\begin{abstract}
Study Design: The study involved the long-term assessment of persons with spinal cord injury (SCI) who previously participated in a nonrandomized longitudinal controlled trial.

Objectives: The objective was to report on the effectiveness of early psychological intervention in improving some aspects of adjustment to SCI 2 years post injury.

Setting: The study was conducted in Sydney, Australia.

Methods: Twenty-eight SCI persons who had participated in group Cognitive Behaviour Therapy (CBT) during hospital rehabilitation were assessed for drug usage, hospital readmissions, relationships, perceived adjustment and social discrimination 2 years after treatment. The intervention group's responses on the measures were compared with a control group of 31 SCI persons who only received traditional rehabilitation services during their hospitalization.

Results: Subjects in the treatment group experienced less hospital re-admissions, used less drugs and reported higher levels of adjustment compared to the control group. Relationships were stable for both groups and there were no suicides in either group up to 2 years. There were no differences in perceived social discrimination between the two groups.

Conclusion: The study suggests the long-term adjustment for SCI persons is encouraging for the majority. However, the provision of group CBT for SCI persons appears to improve significantly some aspects of adjustment to the injury in the long-term.
\end{abstract}

Keywords: adjustment; treatment; SCI; long-term

\section{Introduction}

For equity and humanitarian reasons, it is important that the life of persons suffering traumatic spinal injury (SCI) be improved. Persons with SCI have experienced a major trauma and among other things, must deal with the prospect of being permanently injured, having high levels of dependence and perhaps experiencing significant pain. Controlled research needs to be conducted into long-term SCI outcomes and into methods of improving adjustment to the injury. Information available suggests all is not well longterm in the SCI population. A 9 year longitudinal study of people with $\mathrm{SCI}^{1}$ showed that while employment and financial aspects were enhanced, well-being decreased over the 9 year period. Well-being and adjustment were measured by self-report questionnaire, and included factors like adjustment, satisfaction with family relationships and social life, sexual activity, discrimination, depression, pain and feelings of dependency. Furthermore, suicide rates amongst persons with SCI may be four to five times that of the age-sex-race-specific rates for the general population $^{2,3}$ and psychological factors are most likely

*Correspondence: Dr A Craig, Department of Health Sciences, University of Technology, PO Box 123, Broadway, 2007, Sydney, Australia related. ${ }^{4}$ Research ${ }^{5}$ has shown that mortality rates of SCI persons are significantly higher for diseases like septicemia (82.2 times more likely), pneumonia (37.1 times) and pulmonary emboli (46.9 times more likely). Prevalence of self-neglect (such as non-compliant behavior or passive behavior resulting in physical problems) has also been found to be high when depressive illness is involved. ${ }^{6}$ Australian data on divorce trends following SCI is lacking, however, increased divorce trends in the USA demonstrate the difficulties involved in adjusting to SCI. ${ }^{7}$ Drug abuse is also a potentially increasing problem in this population. ${ }^{8}$

The Sydney Spinal Cord Injury Study (SSCIS), ${ }^{9-13}$ a nonrandomized controlled trial, has been in progress since 1989 and has endeavored to scientifically evaluate aspects of adjustment to SCI. In the first stage, the study concentrated upon attitudinal aspects of adjustment including anxiety, depressive mood, selfesteem, locus of control and coping styles. Results showed that about $30 \%$ of SCI persons have significantly raised levels of anxiety, depressive mood and feelings of helplessness in comparison to age, education and sex matched non disabled controls., ${ }^{9}, 10$ It was a matter of concern that these raised levels did not diminish over time up to 2 years. ${ }^{9,10}$ However, SCI 
persons were shown to have similar levels of selfesteem and positive coping styles to the non disabled controls. ${ }^{10}$ In a second stage of the SSCIS, cognitive behavior therapy (CBT) delivered in the rehabilitation phase, was shown to reduce depressive mood and anxiety to normal levels in depressed and anxious SCI persons immediately after treatment and this was maintained up to 2 years after the injury. ${ }^{11,12} \mathrm{SCI}$ persons with high levels of depressive mood who participated in the first stage and who did not receive this therapy, maintained moderate to high levels of anxiety and depressive mood up to 2 years after the injury. ${ }^{11,12}$ Furthermore, feelings of helplessness were significantly reduced in SCI persons treated with CBT compared to the controls. ${ }^{13}$

CBT was used as it is increasingly being recognized as an effective technique for managing a wide range of disorders, in particular depression and anxiety. ${ }^{14}$ It is a structured and effective therapeutic approach which attempts to change behavior and thinking patterns that are believed to be associated with the problem behaviour and/or emotions. Therapists utilizing this approach attempt to change behavior and feelings by manipulating behavioral contingencies (for example, implementing rewards), isolating thought processes needed to be changed (such as negative or irrational thoughts) and teaching strategies of generating rational and positive thoughts. CBT may psychologically prepare SCI persons for the physical and social barriers they may encounter in the community, possibly preventing the onset of depression and anxiety that can be associated with the injury.

The purpose of this research was to report further on some aspects of adjustment 2 years after the injury. That is to determine whether CBT reduced hospital readmissions, improved relationships, reduced drug usage, and improved perceived social discrimination and self-reported perceptions of adjustment in the intervention group compared to the controls.

\section{Methods}

\section{Participants}

Treatment group Participants consisted of SCI persons consecutively admitted to a SCI ward over a 2 year period $(1991-1992)$. Only subjects who were newly injured, had permanent neurological loss, had no head injuries or any pre-existing psychopathology (as determined by the nursing unit manager's or medical officer's report), were at least 16 years of age, and able to speak English were selected for the study. The treatment group consisted of 23 males and 5 females with a mean age of 31 years, ranging from $16-58$ years $(\mathrm{SD}=11.8)$. There was an attrition of one subject at the 2 year assessment, resulting in a final sample of 27 subjects 2 years post-injury. Forty-six per cent of the group were diagnosed as paraplegic and $54 \%$ as quadriplegic. There were no significant differences in demographics between those with paraplegia and those with quadriplegia ${ }^{9}$ so the two groups were combined as one sample for this study. The majority of subjects $(71 \%)$ had complete lesions, while $21 \%$ were incomplete. All injuries but one were traumatic in nature.

Control group Subjects included persons sustaining an acute SCI admitted to the teaching hospital acute SCI wards from 1989-1990 and who were participants in research reported in this journal. ${ }^{9}$ Selection criteria were the same as for the treatment group. The final sample up until 1 year post-injury consisted of a total of 41 subjects. However, there was an attrition of ten subjects for the 2 year data, as a result of difficulties in locating subjects, failure to return the questionnaires and death in the case of one person (due to a cardiac arrest). Therefore 31 control SCI subjects participated in the complete study. As far as the researchers are aware, none of the sample who did not respond at the 2 year follow-up had committed, or attempted to commit suicide. The control group were not significantly different to the treatment group in terms of demographics with $51 \%$ diagnosed as paraplegic and $49 \%$ as quadriplegic. The majority of subjects $(68 \%)$ has complete lesions, while $32 \%$ were incomplete. No significant differences were found for site of lesion or completeness of break in terms of demographics or overall scores in the psychological measures, so the controls were combined as one sample. All injuries were traumatic in nature. The controls received traditional rehabilitation services only. Around half of the controls were selected from the second SCI ward not involved in the CBT treatment mentioned below. Rehabilitation and medical treatment delivered in the two hospitals was very similar at the time of the study. Furthermore, there were no significant differences in demographics or personality between the controls from either hospital $^{9-11}$ so the two groups of controls were combined as one sample for this study.

Treatment Treatment was conducted at one of the two acute SCI wards in Sydney, Australia. The program was conducted over 10 weeks in small groups (an average of four to five persons per group) for 1.5$2 \mathrm{~h}$ per week, replacing normal rehabilitation therapy. CBT attempts to change behavior and feelings that are believed associated with the problem and which are considered maladaptive. A group CBT approach was employed, including components that addressed anxiety, depression, self-esteem, assertion, sexuality and family relations. The aim of the program was to provide cognitive and behavioral skills to cope with the psychological and social difficulties encountered upon entering the community. Components of the program included relaxation techniques (progressive muscle relaxation, visualization techniques, and self-hypnosis); cognitive-restructuring (attention-distraction and pain-reinterpretation techniques, positive reappraisal and positive coping self-statements such as 'Just as I 
have learned to live with SCI, I can learn to live with my pain'); increasing levels of pleasant events; social skills and assertiveness training (role plays and videofeedback to practise assertiveness skills); sexuality sessions including both education and discussions of sexual alternatives and typical problems encountered regarding sexuality and SCI (eg, body image, sexual capabilities), and how to effectively deal with sexuality issues when they occur. On average, SCI subjects attended the majority of treatment sessions. However, if a subject missed a session, the therapist usually arranged an alternative time to individually present the therapy. Each week the therapist allocated time in the session to discuss the subject's use of the skills in the prior week and to encourage adherence. It is important to mention that control subjects (no CBT) received 2 extra hours of physical and occupational therapy a week instead of the CBT. Therefore, the CBT subject did not receive extra therapy time.

Measures All subjects were comprehensively and longitudinally assessed as part of the controlled trial. ${ }^{11-13}$ However, this paper will only report measures taken 2 years after the injury. In order to measure some aspects of adjustment, a brief questionnaire was designed (see Appendix 1) and was comprised of Likert-type and open ended items. To enhance reliability and content validity, all terms used in the questionnaire were fully explained to the SCI subjects. To evaluate the test-retest reliability of the above questionnaire, a group of ten SCI persons in the study were randomly selected and tested 1 week apart. Their answers showed little variability over the 1 week, with the respondents showing at least a $96 \%$ agreement between the two assessments for all the items. In addition, aspects of the questionnaire were validated. For instance, self-reported perceptions of adjustment was validated by subjects completing the Beck Depression Inventory. ${ }^{15}$ The BDI is a well-validated self-report measure of depressive mood. The BDI has been shown to be a suitable and a sensitive instrument for detecting the presence of depression in SCI persons. ${ }^{9}$ Re-admissions were validated by accessing hospital records. In an attempt to validate self reported relationships and drug usage, the interviewer assessed family or friends and confirmed the responses of the SCI subjects.

\section{Results}

\section{Re-admissions}

Subjects were asked whether they had been re-admitted to hospital following discharge. Almost six out of ten of the control subjects $(n=17,55 \%)$ and four out of ten $(n=11,41 \%)$ of the treatment group had been readmitted. The difference in group re-admission rates was significant $\left(\chi^{2}=3.93, P<0.05\right)$, with the treatment group having fewer re-admissions than the controls. Four $(13 \%)$ of the controls and $2(6 \%)$ of the treatment group had been re-admitted more than once. The most common reasons for re-admission were pressure areas $(n=4$ for controls, $n=2$ for treatment group) and urinary tract infections $(n=3$ for controls and $n=6$ for treatment). This self-report by participants of their re-admission was validated objectively by evaluating a random selection of ten of the subjects' medical records. These were checked against their self-reports of re-admission rate, with only one person stating that they had not been readmitted when in fact Medical Records showed they had.

\section{Drug usage}

Subjects were asked whether they believed they used any of the following drugs at the time of assessment or since discharge: 'cigarettes', 'hallucinogens (eg, marijuana)', 'antidepressants', 'anti-anxiety drugs', 'pain killers', 'sleeping tablets' or 'alcohol'. Approximately $40 \% \quad(n=12)$ of the controls said that they frequently used one of the above drugs. Four subjects $(13 \%)$ reported that they frequently used pain killers, two $(7 \%)$ frequently used cigarettes, one claimed to frequently use sleeping tablets, and one hallucinogens. An additional three subjects chose more than one category. Four $(15 \%)$ of the treatment group claimed to frequently use any of the drugs listed, with two $(7.5 \%)$ using painkillers and two (7.5\%) sleeping tablets. The controls were found to have a significantly higher frequency of self-reported drug usage compared with the treatment group $\left(\chi^{2}=4.12\right.$, $P<0.05)$. Excluding cigarettes, $29 \%$ of the controls and $15 \%$ of the treatment group reported that they frequently used antidepressants, anxiolytics, hallucinogens, painkillers or sleeping tablets. As far as possible, the researchers were confident the data on drug usage was accurate as available family and friends were consulted on the drug usage of the SCI participants.

\section{Relationships}

Four categories (Table 1) were used to determine relationship outcomes over the 2 years. Of the $42 \%$ $(n=13)$ of the control group who had been in a stable relationship prior to their injury, only three persons had split from their partner. Rates were similar for the treatment group, with four out of 14 (52\% having a stable relationship prior to the injury) having split from their partner since the injury $(P=\mathrm{NS})$. Of those who had no pre-injury relationship, around four out of ten in the control group and six out of ten in the treatment group had formed new relationships since their injury $(44 \%$ of controls, $61.5 \%$ of treatment group), though this difference was not significant. The researchers were confident the data on relationships was accurate as available family and friends confirmed that the SCI authentically reported their relationships. 
Table 1 Showing marital relationship status 2 years post-injury

\begin{tabular}{|c|c|c|c|c|}
\hline \multirow[b]{2}{*}{ Relationship status } & \multicolumn{2}{|c|}{ Control group } & \multicolumn{2}{|c|}{ Treatment group } \\
\hline & $\mathrm{n}=31$ & $\%$ & $\mathrm{n}=27$ & $\%$ \\
\hline Pre-injury relationship and stable & 10 & 32 & 10 & 37 \\
\hline Pre-injury relationship and split since SCI & 3 & 10 & 4 & 15 \\
\hline $\begin{array}{l}\text { No pre-injury relationship but formed new } \\
\text { relationship since SCI }\end{array}$ & 8 & 26 & 8 & 30 \\
\hline $\begin{array}{l}\text { No pre-injury relationship not formed new } \\
\text { relationship }\end{array}$ & 10 & 32 & 5 & 18 \\
\hline
\end{tabular}

\section{Social discrimination}

Almost half of the controls $(n=15,48 \%)$ and one-third of the treatment group $(n=10,37 \%)$ reported that they never felt discriminated against. These group differences were not significant. However, 14 controls $(n=45 \%)$ and 14 treatment subjects $(n=52 \%)$ felt negatively discriminated against sometimes. Therefore around half of both groups believed that they had experienced a negative social discrimination at some time in the 2 years since their release from hospital.

\section{Self-reports of adjustment}

Subjects were asked how well they thought they had adjusted to living with SCI. Thirteen control subjects $(43 \%)$ reported they had not adjusted well (score of 1 or 2 on the adjustment scale, see Appendix 1), while the remainder (17 or $57 \%$ ) believed they had adjusted moderately to very well (score 3,4 or 5 on the Adjustment scale, see Appendix 1). One subject failed to respond. Only one person (4\%) from the treatment group reported they had not adjusted very well, while the remainder believed they had adjusted moderately to very well $(n=26,96 \%)$. Table 2 shows the adjustment breakdown between the two groups. A Chi square analysis showed the treatment group to have a significantly higher proportion of persons who believed they had adjusted moderately to extremely well $\left(\chi^{2}=12.0, P<0.01\right)$. The self-reported adjustment measure was shown to be valid by calculating Pearson correlations between the self-reported adjustment scores and depressive mood (BDI) scores. Significant negative correlations were found between the groups' self-reported adjustment and their levels of depressive $\operatorname{mood}(r=-0.81$ for the controls and $r=-0.46$ for the treatment group; $P<0.01)$. Subjects who rated themselves high on adjustment were those who more likely score lower on the BDI.

\section{Conclusions}

The findings of the Sydney Spinal Cord Injury Study provide positive information concerning the long-term impact of a specialized CBT program on aspects of adjustment to SCI. Receiving CBT in the rehabilitation phase has previously been reported to reduce depressive mood, anxiety and feelings of helpless-
Table 2 Showing frequency breakdown of perceived adjustment

\begin{tabular}{lcc}
\hline Adjustment & $\begin{array}{c}\text { Control } \\
\text { group }\end{array}$ & $\begin{array}{c}\text { Treatment } \\
\text { group }\end{array}$ \\
\hline Not very well & 13 & 1 \\
Moderately well to very well & 17 & 26 \\
\hline
\end{tabular}

$\chi^{2}=12.0, \mathrm{df}=1, P<0.01$

ness. ${ }^{1-13}$ In addition to these findings, the data presented in this paper suggest CBT is also associated with significantly less hospital re-admissions, less drug usage, and higher levels of self-reported adjustment than the control SCI subjects. There was a non significant trend (though almost a 20\% greater proportion) for the treatment group to have a higher percentage of forming new relationships since the injury than the control group. A power problem due to the low subject numbers is likely to be the reason for the non significance. There were also no differences in reported rates of negative social discrimination and splits in relationships since the injury. No persons in either group committed suicide.

Re-admission to hospital for SCI complications can be a frequently occurring problem for those living with SCI. A re-admission would be disruptive to social, personal and vocational activities, and further depletes an already pressured health dollar. Any strategy that would reduce the re-admission rate should be evaluated and incorporated into ongoing health care. Specialized CBT offered in the rehabilitation phase resulted in almost 15\% less re-admissions than those who receive standard rehabilitation services. Research $^{16}$ has shown the cost of SCI during the 2 years after the injury to be $\$ 22$ million in 1992 US dollars value. Much of this cost was due to physical complications and re-admissions. With pressure on the health dollar occurring in most countries, any strategy that can improve services by reducing health care costs must be utilized. From the results of this current study, the provision of specialized programs in the rehabilitation stage will more than likely contribute to reduced health care costs in the SCI population.

Drug usage can be a major factor contributing to lowered quality of life and poor adjustment. The 
treatment group had $25 \%$ less drug usage than the controls which suggests that the CBT was effective in reducing vulnerability to drug usage. On the positive side, the majority of those surveyed in both groups did not believe they frequently used drugs. However, it is a concern that around $40 \%$ of the controls reported they frequently used drugs and at least $20 \%$ reported they frequently used pain killers or more than one type of drug. Pain has been shown to be a predictor of longterm depression, ${ }^{17}$ and to be related to prevalence of depression and emotional stress. ${ }^{18}$ Therefore, more emphasis should perhaps be placed upon the use of non pharmacological pain management techniques during the rehabilitation phase in hospital.

Findings were positive for relationships. The majority of those who were married remained so up to 2 years post-injury, and approximately half of those who were not married had formed new relationships since their injury, though the difference was not significant. There was a trend in the treatment group to be more likely to form new relationships since the injury than those in the control group. However, it was concerning to find over $50 \%$ of the subjects, regardless of group, had experienced some form of negative social discrimination. This remains a problem for persons with SCI, and needs to be dealt with on two levels. First, SCI persons need to be offered support and assertiveness training that would strengthen their selfesteem and self-concepts. They could then better manage discrimination when it occurs. Second, society needs to be better educated and informed about the nature of the injury and the needs of the SCI persons.

CBT was associated with higher levels of selfreported adjustment. Sixty-three per cent of the treatment group said that they had adjusted either very well or extremely well. However, 2 years after the injury, almost $50 \%$ of the controls were not satisfied with their adjustment. These perceptions of adjustment were validated by the significant correlations with scores on the Beck Depression Inventory. Those who believed they had adjusted well tended to have lower depressive mood scores than those who believed they had not adjusted well. Mean depressive mood scores 2 years post-injury ${ }^{9}$ suggest SCI persons are prone to suffer mild to moderate levels of depression, which researchers have shown do not necessarily decrease with time. ${ }^{9}$ In the absence of specialized treatment like CBT, high levels of depressive mood may well persist in many SCI persons up to 2 years making adjustment to the disorder very difficult. ${ }^{11}$ Further research should investigate whether persistent depression leads to greater risks of suicide, re-admissions, diseases and self-neglect. Health professionals involved with SCI persons will need to be aware of this possibility. However, it must also be remembered that not all SCI persons suffer significant depression and anxiety in the long-term. Therefore, it is imperative to be able to differentiate between those who are at risk and those who are not at risk of remaining depressed or anxious in the long-term.
While the results suggest CBT is beneficial, some possible design difficulties must be discussed. First, subject numbers were low, however, this is more a problem for rejecting correctly the null hypothesis or finding true differences (a Type II error occurs when the null is falsely retained). If differences with low subject numbers are found between the CBT and control groups, this suggests the differences (effect sizes) are large and therefore clinically meaningful. Second, increased attention due to the CBT could have accounted for the improvements seen in the CBT subjects relative to the controls. However, the controls did not receive a 'waiting list' placebo. All controls received the equivalent number of therapy hours to the CBT group. The CBT group received $2 \mathrm{~h}$ less a week of the standard rehabilitation therapy. Therefore, the differences found are unlikely to have been due to increased attention. The researchers believe the specific influence of CBT accounted for much of the change. The researchers are currently conducting a comprehensive study of adjustment on the above SCI subjects 8 years after the initial injury. It is hoped this data will further enhance our understanding of long-term adjustment to SCI and further clarify the influence of a structured treatment like CBT.

\section{Acknowledgements}

This study was supported by a grant from the Research and Development Grants Advisory Committee (RADGAC now NHMRC), Motor Accident Authority grant, University of Technology, Sydney grant and Department of Community Services and Health, Canberra, Australia. Thanks for assistance to the Staff of the spinal units of Prince Henry and Royal North Shore Hospitals Sydney, in particular Sister Margaret Samuels, Mr Glen Stolzenheim, Professor Richard Jones, Dr Sue Rutkowski and Dr John Yeo.

\section{References}

1 Krause JS. Adjustment after spinal cord injury: A 9-year longitudinal study. Arch Phys Med Rehabil 1997; 78: 651 - 657.

2 De Vivo MJ, Black KJ, Richards S, Stover SL. Suicide following spinal cord injury. Paraplegia 1991; 29: 620-627.

3 Harris EC, Barraclough BM. Suicide as an outcome for medical disorders. Medicine 1994; 73: $281-296$.

4 Krause J, Crewe N. Prediction of long-term survival among persons with spinal cord injury: an 11 year prospective study. Rehabil Psychol 1987; 32: $205-213$.

5 De Vivo MJ, Black KJ, Stover SL. Causes of death during the first 12 years after spinal cord injury. Arch Phys Med Rehabil 1993; 74: $248-254$.

6 Macleod AD. Self-Neglect of Spinal Injured Patients. Paraplegia 1988; 26: $340-349$.

7 De Vivo MJ, Fine PR. Spinal cord injury: Its short-term impact on marital status. Arch Phys Med Rehabil 1985; 66: 501-504.

8 Bedbrook G, Beer NE, McLaren RK. Preventative measures in the tertiary care of spinal cord injured people. Paraplegia 1985; 23: $69-77$.

9 Craig AR, Hancock KM, Dickson H. A longitudinal investigation into anxiety and depression over the first two years of spinal cord injury. Paraplegia 1994; 32: 675-679. 
10 Craig AR, Hancock K, Chang E. The influence of spinal cord injury on coping styles and self-perceptions two years after the event. Aust New Zeal J Psychiatry 1994; 28: 307-312.

11 Craig A, Hancock K, Dickson H, Chang E. Long-term psychological outcomes in spinal cord injured persons: Results of a controlled trial using cognitive behavior therapy. Arch Phys Med Rehabil 1997; 78: $33-38$.

12 Craig A, Hancock K, Chang E, Dickson H. Immunizing against depression and anxiety after spinal cord injury. Arch Phys Med Rehabil 1998; 79: 375-377.

13 Craig A, Hancock K, Chang E, Dickson H. The effectiveness of group psychological intervention in enhancing perceptions of control following spinal cord injury. Aust New Zeal J Psychiatry 1998; 31: $112-118$.
14 Andrews G. The essential psychotherapies. Brit J Psychiatry 1993; 162: $447-451$.

15 Beck AT et al. An inventory for measuring depression. Arch Gen Psych 1967; 4: $351-363$.

16 Johnson RL, Brooks CA, Whiteneck GG. Cost of traumatic spinal cord injury in a population-based registry. Spinal Cord 1996; 34: $470-480$.

17 Craig AR, Hancock KM, Dickson H. Spinal cord injury: A search for determinants of depression two years after the event. Brit J Clin Psychol 1994; 33: 221 - 230.

18 Anke AGW, Stenehjem AE, Stanghelle JK. Pain and life quality within 2 years of spinal cord injury. Paraplegia 1995; 33: $555-$ 559.

Appendix 1 Questionnaire developed for the study

1 Since your discharge, have you been re-admitted. How many times?

2 Circle those items that were the reasons for re-admission
(a) Urinary tract infection or other kidney problem
(b) Pressure area
(c) Respiratory problems (eg pneumonia)
(d) Surgery (eg spinal, suprapubic catheter)
(e) Other

3 Please circle any of the following you have used since discharge from hospital
(a) Alcohol
(b) Hallucinogens (eg marijuana)
(c) Antidepressants
(d) Anti-anxiety drugs
(e) Pain killers
(f) Cigarettes
(g) Sleeping tablets

4 If you were married/defacto before your injury, have you split from your partner since your injury?
(a) Yes
(b) No
(c) Not applicable

5 If you did not have a partner before your injury, have you been able to form a new relationship since discharge from hospital?
(a) Yes
(b) No
(c) Not applicable

6 Do you feel discriminated against socially (eg people treating you as though you were intellectually disabled?)
1
Almost never Sometimes Often
Almost always

7 How well do you think you have adjusted to having a spinal injury? Not at all well
Not very well
Moderately well
Very well
5
Extremely well 